\title{
La autoeficacia como recurso en el proceso de socialización laboral Una propuesta de intervención
}

\author{
Ana López Ramos \\ anapositivat@gmail.com \\ Isabel Maria Martínez Martínez \\ imartine@uji.es
}

\section{Resumen}

Actualmente hay un gran número de empresas que se están dando cuenta de la importancia de gestionar correctamente la incorporación de nuevos empleados en sus plantillas. $Y$ es que este proceso es de una gran complejidad y puede tener fuertes repercusiones tanto en la salud psicosocial de los trabajadores como en la salud financiera de la organización. Consideramos que la autoeficacia de los nuevos empleados puede estar jugando un papel fundamental en la adaptación de estos a su nuevo entorno. Es por ello que el objetivo de este programa es utilizar la autoeficacia como elemento central para facilitar la incorporación de nuevos trabajadores a la organización. Para ello, se realizarán cuatro talleres que incidirán en las cuatro fuentes principales de autoeficacia. Los primeros tres talleres se realizarán previamente a la incorporación de los nuevos trabajadores: 1) el diseño de tareas de menor a mayor dificultad, incidiendo en las experiencias de éxito; 2) la realización de un programa mentoring, que trata de actuar sobre el aprendizaje vicario; 3) la programación de reuniones de feedback y refuerzo, actuando de esta forma en la persuasión social. El último taller se realizará tras la incorporación de los nuevos trabajadores y consistirá en unas jornadas de bienvenida y en varias actividades de mindfulness, con lo que se pretenden facilitar los indicadores fisiológicos de calma y relajación. El éxito de este programa se basa en el abordaje de todas las fuentes de la autoeficacia, con la intención de crear sinergias entre estas y favorecer así la consecución de los objetivos de la intervención. Creemos que este tipo de programa puede reducir el estrés asociado a la incorporación al mercado laboral, especialmente cuando los trabajadores son recién titulados. Además, por la literatura existente, consideramos que existen evidencias suficientes para hipotetizar que el incremento en la autoeficacia laboral provocará, a su vez, el aumento del engagement y del bienestar en el trabajo, con lo que se utilizarán distintos cuestionarios para medir dichos constructos antes y tras la intervención.

Palabras clave: autoeficacia, inserción laboral, socialización, engagement, bienestar. 


\begin{abstract}
A large number of companies are now realising the importance of properly managing the incorporation of new employees into their workforce. This process is highly complex and can have a strong impact on the psychosocial health of workers and the financial health of the organisation. We believe that the selfefficacy of new employees may be playing a key role in their adaptation to their new environment. That is why the aim of this programme is to use self-efficacy as a central element to facilitate the incorporation of new workers into the organisation. To this purpose, four workshops will be held that will focus on the four main sources of self-efficacy. The first three workshops will be held prior to the incorporation of new workers: 1) the design of tasks from least to most difficult, focusing on successful experiences; 2) the implementation of a mentoring program, which seeks to act on vicarious learning; 3 ) the scheduling of feedback and reinforcement meetings, thus acting in social persuasion. The last workshop will take place after the incorporation of the new workers and will consist of welcome days and various mindfulness activities, which are intended to facilitate physiological indicators of calm and relaxation. The success of this programme is based on addressing all sources of self-efficacy, with the intention of creating synergies between them, and thus promoting the achievement of the objectives of the intervention. We believe that this type of program can reduce the stress associated with entering the labor market, especially when workers are newly qualified. In addition, from the existing literature, we consider that there is sufficient evidence to hypothesize that the increase in labor self-efficacy, in turn, will provoke an increase in engagement and well-being at work, with which different questionnaires will be used to measure these constructs before and after the intervention.
\end{abstract}

Keywords: self-efficacy, job placement, socialization, engagement, well-being.

\title{
Introducción
}

La inducción de nuevas personas en una organización nunca es un proceso sencillo. En esta transacción se ven involucrados muchos factores, tanto individuales como de la propia organización, y se tiene que actuar con sumo cuidado para evitar que haya un mal ajuste entre la persona y su nueva posición dentro de la organización.

Las consecuencias emocionales que supone un mal ajuste del trabajador pueden ser nefastas para la persona pero, sobre todo, para la organización. El empleado puede manifestar un descenso en su compromiso con la organización, empezar a desarrollar conductas agresivas $u$ hostiles hacia la organización o sus compañeros e incluso incrementar su intención de abandono (Mendoza 2003). Esto se traduce en una menor productividad, una mayor rotación del personal y un gran perjuicio para la imagen empresarial (Reyes y Martínez 2007). Según un estudio realizado por Bersin Deloitte's, hay un $4 \%$ de los empleados que abandonarán la empresa si tienen un primer mal día, además, un $22 \%$ de la rotación del nuevo personal se producirá en los primeros 45 días. Las consecuencias económicas de esta rotación inicial del personal será de al menos tres veces más que el sueldo de un empleado (Bersin Deloitte's 2014).

Por otro lado, la realidad laboral actual requiere que las organizaciones cuenten con una fuerza laboral que, además de los conocimientos y habilidades necesarias para 
desempeñar sus tareas, esté comprometida con la corporación, sea proactiva, innovadora, resiliente... y un sinfín más de características (Fisher 1986). La organización, por lo tanto, debe proveer de recursos a sus trabajadores, no solo para atraer al talento, sino también para conservarlo. Afortunadamente, las empresas empiezan a darse cuenta de la importancia del proceso de socialización. La socialización en la empresa se entiende como «un proceso de aprendizaje mediante el cual se adquieren las actitudes, habilidades y conductas útiles para el desempeño del trabajo» (Peiró y Prieto 1996).

Tradicionalmente, el proceso de socialización se divide en tres fases: 1) la fase de anticipación, 2) la fase de encuentro y 3) la fase de cambio o adquisición. En la fase de encuentro es donde se realizan las actividades de socialización propiamente dichas. Por regla general, todos los nuevos trabajadores sufren una conmoción ante la realidad al entrar a formar parte de la organización e intentan reducir la incertidumbre buscando información de su ambiente. Es en este momento cuando la empresa puede favorecer la adaptación del nuevo empleado brindándole la información necesaria para reducir la incertidumbre. Estas técnicas pueden ser bien técnicas individuales o bien institucionales o colectivas (Jones 1986).

Un hito fundamental que no podemos olvidar en el proceso de socialización del personal es la gestión del contrato psicológico. El contrato psicológico se considera como las percepciones presentes entre trabajador y organización, que tienen naturaleza laboral, e implica las obligaciones implícitas entre ellos (Tena 2002). De estas percepciones iniciales se generarán una serie de expectativas entre ambos y es fundamental buscar un buen ajuste entre las expectativas del empleado y la realidad laboral, para evitar la conocida violación del contrato psicológico, que se produce cuando se percibe que una de las partes no está cumpliendo con sus obligaciones. La rotura de este contrato psicológico puede provocar emociones y conductas negativas $y$, además, es posible que nunca se logre recuperar la confianza del trabajador (Robinson y Rousseau 1994).

Como podemos observar, la socialización laboral es un proceso bastante complejo y muy sensible, y la organización deberá apostar los recursos necesarios para asegurar la fidelización del trabajador. No obstante, es posible que, utilizando algunos recursos claves, podamos facilitar dicho proceso y reducir los costes que ocasiona. Una línea de actuación estratégica podría ser utilizar los recursos personales del trabajador. Uno de los recursos personales más investigados y valorados en el ámbito organizacional es la autoeficacia.

La autoeficacia se enmarca como elemento central de la teoría del aprendizaje social de Albert Bandura y se refiere a «las creencias de la gente en sus propias capacidades para movilizar la motivación, los recursos cognitivos y el curso de acción necesarios para ejercer control sobre los acontecimientos de sus vidas» (Wood y Bandura 1989). La autoeficacia afecta a: 1) nuestro comportamiento, mediante la elección de conductas, ya que la persona escogerá aquellas pautas de acción en las que cree que tendrá éxito; 2) nuestro pensamiento, amplificando o reduciendo las demandas del entorno dependiendo de si nos sentimos eficaces o no para afrontarlas; 3) nuestros sentimientos, provocando bien estados de ansiedad o estrés o bien estados de relajación y control (Martínez-Martínez y Salanova-Soria 2006).

Según Bandura (2001), existen cuatro fuentes fundamentales de autoeficacia sobre las que podemos intervenir:

1. Experiencias de éxito: esta es la fuente principal de autoeficacia. Mediante la acumulación de logros el empleado va construyendo sus creencias de eficacia. Esto ocurrirá siempre y cuando el trabajador perciba que la tarea era retadora y ha 
realizado un esfuerzo y que el éxito es el resultado de su esfuerzo y no de factores externos.

2. Aprendizaje vicario: mediante la observación de los éxitos de otro sujeto pueden incrementar la autoeficacia del observador. Esto va a depender de la similitud percibida por el trabajador, es decir, que perciba que sus capacidades o características son similares a las del modelo.

3. La persuasión verbal: que haría referencia al feedback que se le proporciona al sujeto respecto a su ejecución. Dependerá de la percepción que tenga la persona respecto al informador, si este es una persona valorada, de confianza para el sujeto o que suponga una autoridad (por ejemplo, un supervisor).

4. Estados fisiológicos o activación emocional: los indicadores fisiológicos de calma o control incrementarán la autoeficacia del sujeto, mientras que los estados de ansiedad o estrés la reducirán.

Hay multitud de estudios sobre la autoeficacia en el ámbito laboral que relacionan esta con diversos constructos. Por ejemplo, en un estudio realizado por Consiglio, Borgogni, Alessandri y Schaufeli (2013) se demostró que la autoeficacia laboral era un importante antecedente del desempeño laboral y el comportamiento cívico organizacional. Esto está muy relacionado con las investigaciones que muestran el papel mediador de la autoeficacia con el engagement tanto profesional (Lisbona, Palaci, Salanova y Frese 2018) como académico (Pérez-Fuentes et al. 2018). Otro elemento que se ha relacionado con la autoeficacia es el bienestar psicológico. La autoeficacia puede tener un papel amortiguador del estrés y, por lo tanto, de los efectos negativos de este (Calderón, Laca y Pando 2017). Según el modelo espiral dual de la salud ocupacional, desarrollado por Salanova et al., el recurso personal de autoeficacia provocaría que las demandas fueran percibidas como retadoras, a la vez que se percibirían más recursos, lo que aumentaría el bienestar psicosocial y, por lo tanto, las consecuencias organizacionales, dando lugar a espirales positivas de motivación (Salanova, Llorens y Schaufeli 2011).

Vistos los beneficios que tiene dicho constructo en el contexto laboral, la presente propuesta de intervención pretende combinar las distintas fuentes de autoeficacia para incrementar la autoeficacia laboral de los empleados durante su proceso de incorporación a la organización. Se espera que la inversión en recursos personales realizada por la organización aumente, además de la autoeficacia, el bienestar y el compromiso de los nuevos trabajadores. De esta forma se pretende reducir el impacto emocional negativo asociado a la inducción, evitando así efectos negativos como la fuga del talento y la aparición de estados psicológicos positivos (engagement).

\section{Objetivo e hipótesis}

El objetivo del presente estudio es mostrar una intervención sobre la autoeficacia laboral consistente en cuatro talleres que inciden en las cuatro principales fuentes de autoeficacia: experiencias de éxito, persuasión social, aprendizaje vicario e indicadores fisiológicos de calma y control.

Derivado de la intervención, se hipotetiza que:

- $\mathrm{H}_{1}$ : La autoeficacia laboral de los nuevos trabajadores incrementará posteriormente a la intervención realizada.

Al mismo tiempo, de la revisión de la literatura científica, se forman las siguientes hipótesis:

- $\mathrm{H}_{2}$ : El aumento de la autoeficacia laboral provocará, a su vez, un incremento en el engagement laboral de los nuevos trabajadores. 
- $\mathrm{H}_{3}$ : El aumento de la autoeficacia laboral provocará, a su vez, un incremento en el bienestar laboral de los nuevos trabajadores.

- $\mathrm{H}_{4}$ : El aumento de la autoeficacia laboral provocará, a su vez, un incremento en el desempeño y la productividad de los nuevos trabajadores.

Para evaluar el efecto de la intervención se utilizarán cuestionarios específicos para cada uno de los constructos, además de los datos de desempeño de los que disponga la organización.

\section{Método}

\section{Muestra}

En relación con las necesidades de la organización, se requiere la incorporación de 25 profesionales que irán destinados a los departamentos de vigilancia de la salud, técnicos superiores de las tres especialidades de prevención de riesgos (seguridad, higiene industrial y ergonomía y psicosociología) y administrativos. Por lo tanto, la muestra está constituida por 25 trabajadores/as, cuyas edades oscilan entre los 25 y 30 años, siendo la muestra paritaria respecto al género de los mismos.

\section{Evaluación}

- Autoeficacia. Respecto a la autoeficacia profesional, será evaluada con el cuestionario de autoeficacia profesional (AU-10). Este es un instrumento de autoinforme compuesto por 10 ítems que evalúan las creencias de los empleados para realizar con éxito su labor profesional pese a la aparición de obstáculos, con una escala tipo Likert de siete puntos, de 0 («incapaz de poder hacerlo») hasta 6 («seguro de poder hacerlo»). Un ejemplo de ítem de autoeficacia profesional es «seré capaz de encontrar lo que quiero en mi trabajo aunque alguien se me oponga» (Maffei, Spontón, Spontón, Castellano y Meandro 2012).

- Engagement. Será evaluado mediante el Utrecht Work Engagement Scale (UWES). Este instrumento fue desarrollado por el equipo de Schaufeli y tiene diferentes versiones. La utilizada en este proyecto tiene 17 ítems que evalúan las tres dimensiones del engagement: vigor ( 6 ítems) («En mi trabajo me siento lleno de energía»), dedicación (5 ítems) («Estoy entusiasmado con mi trabajo») y absorción (6 ítems) («Soy feliz cuando estoy absorto en mi trabajo»); medido con una escala tipo Likert de siete puntos, comprendidos desde el 0 («nunca, ninguna vez») hasta el 6 («siempre, todos los días») (Schaufeli y Bakker 2003).

- Bienestar. Será evaluado mediante el cuestionario de bienestar laboral teneral (qBLG), que estima las dimensiones psicológicas del bienestar en el entorno laboral. El cuestionario tiene dos dimensiones principales: el bienestar psicosocial, compuesto por la escala de afecto, competencias y expectativas (ejemplo de ítem: "Actualmente en mi trabajo me siento inseguro/seguro»), y efectos colaterales, que cuenta con subescalas de somatización, desgaste y alienación (ejemplo de ítem: «Actualmente, por causa de mi trabajo, siento dolores de cabeza»). Dicho cuestionario tiene una escala de medida tipo Likert de siete puntos, que va desde el 1 hasta el 7, donde el 1 es la presencia de emociones negativas, aparición de trastornos psicosomáticos, falta de competencias (por ejemplo, insensibilidad, trastornos digestivos, sobrecarga de trabajo...), mientras que las respuestas cercanas al 7 nos ofrecen indicadores 
de bienestar en el trabajo o inexistencia de síntomas psicosomáticos (satisfacción, esperanza, éxito...) (Blanch, Sahagún, Cantera y Cervantes 2010).

Los tres instrumentos serán administrados en dos tiempos: T1 al final el proceso de selección, tras la comunicación de la contratación al nuevo empleado; y T2 al finalizar el programa de intervención propuesto. Además, se harán dos evaluaciones adicionales de seguimiento, tras los primeros 3 y 6 meses de trabajo en la organización.

- Datos administrativos. Los resultados extraídos con estos tres instrumentos se complementarán con datos administrativos: medidas de desempeño, productividad, rotación del personal y absentismo.

- Entrevista. Al mismo tiempo, al finalizar el proyecto, se realizarán entrevistas individuales para evaluar cualitativamente la satisfacción de los nuevos egresados, la eficacia que perciben que ha tenido el programa y qué factores destacarían y cuáles mejorarían.

\section{Procedimiento}

Como ya se ha hablado en la introducción, se van a utilizar las principales fuentes de autoeficacia (experiencias de logro, aprendizaje vicario, persuasión verbal e indicadores fisiológicos) para facilitar la incorporación de los nuevos empleados a la empresa.

\section{TALLERES PRE-INCORPORACIÓN}

\section{Formación de mentores}

El mentoring es una estrategia muy utilizada cuyo objetivo es facilitar la adaptación a nuevos entornos, que se sirve de la experiencia de otros para reducir el tiempo de aprendizaje (De Miguel, Dema y Pérez 2010). Se entiende como «un proceso de mejora guiado y flexible, con un apoyo continuo, que logra el desarrollo a largo plazo del aprendiz y le capacita para la comprensión de temas personales y organizativos, que pueden afectar el desempeño actual o futuro» (Núñez 2010).

La base de esta estrategia es el aprendizaje vicario, que es una de las fuentes de autoeficacia identificadas por Bandura en su teoría del aprendizaje social (2001). Se basa en el supuesto de que la observación de la ejecución correcta de tareas por el modelo facilitará el aprendizaje del observador. No obstante, el mentor debe poseer unas ciertas características, entre las que destacan la capacidad para transmitir conocimientos y buenas habilidades de comunicación, además de tener una experiencia razonable en la organización (Tristán 2017). Los trabajadores que serán mentores deben representar un verdadero modelo de la conducta deseada. Las actividades relacionadas con este módulo se ordenarán de la siguiente forma:

- Campaña de captación y motivación de mentores. El objetivo es encontrar trabajadores voluntarios y con la disposición suficiente para realizar el programa.

- Conocimientos sobre el programa Mentoring. Se trataría de una sesión donde se abordarán los temas básicos sobre el mentoring

- Habilidades pedagógicas. Durante esta sesión se capacitará a los mentores en competencias de transmisión de conocimientos. 
- Capacidades de comunicación. Se realizarán talleres de comunicación efectiva y asertividad.

\section{Diseño de las tareas}

Con diseño de las tareas nos referimos al denominado diseño del trabajo, que se refiere a la "forma en que se organiza un conjunto de tareas o un trabajo entero» (CCSSO 2002). Como ya hemos comentado en la introducción, las experiencias de éxito son fundamentales en la construcción de la autoeficacia, por lo que el escalamiento de las tareas, teniendo en cuenta el grado de conocimientos/habilidad necesaria para realizarlos, puede ser una técnica crucial para asegurar el éxito del programa. Esta parte del proyecto involucraría especialmente al departamento de Recursos Humanos de la organización, ya que deberían realizar un análisis detallado del puesto y escalar las tareas de menor a mayor complejidad. Además, habrían de determinar el grado mínimo de habilidad demostrada para pasar a la siguiente tarea.

Esta es una actividad especialmente compleja debido a la gran diversidad de empleados nuevos que componen nuestra muestra. No todos los nuevos trabajadores pertenecerán al mismo departamento, por lo que habrá que hacer un proceso de análisis del puesto y su pertinente escalamiento de tareas para cada uno de los puestos que van a ser cubiertos. No obstante, esta parte del programa es crucial, no solo para mejorar la autoeficacia, sino también para garantizar un proceso de selección efectivo, ya que, desglosando el puesto en tareas y competencias, podemos tener una mejor imagen del empleado idóneo.

\section{Refuerzo social}

Esta parte está muy relacionada con las dos anteriores y se refiere a la tercera fuente de autoeficacia: la persuasión verbal. Esta persuasión haría referencia a halagar los esfuerzos del empleado, hacer críticas positivas, reforzar verbalmente los éxitos, dar apoyo en los fracasos... (Schneider 1987). Al finalizar una tarea con éxito, el mentor o el supervisor deberá reforzar el trabajo realizado, dando feedback de la ejecución: aspectos que ha realizado bien, aspectos que se podrían mejorar y aspectos que ha realizado mejor de lo esperado.

Por lo tanto, en el escalamiento de las tareas se deberán intercalar reuniones de feedback entre el mentor y el mentee donde, además de favorecer la crítica constructiva, se refuerce verbalmente al trabajador por sus éxitos y conductas deseables, favoreciendo tanto el desarrollo y el aprendizaje como el aumento de la autoeficacia profesional.

\section{TALLERES POST-INCORPORACIÓN}

\section{Talleres de socialización}

Son actividades dedicadas a transmitir la cultura organizacional. Al inicio se debe dar información suficiente para que el nuevo empleado tenga una visión panorámica de la vida en la organización: valores, misión y visión de esta, políticas de empresa, organigrama y revisión de los procedimientos. Es importante que esta información se plasme en algún tipo de documento que el trabajador pueda repasar en cualquier momento: son los denominados manuales de acogida. Se espera que la familiarización con el ambiente de trabajo reduzca los estados ansiógenos asociados a la incertidumbre. Con los talleres de socialización se pretende aumentar los estados 
fisiológicos de calma y control, que constituyen la cuarta fuente de autoeficacia. EI taller de socialización se realizará en forma de jornadas de bienvenida. El nuevo trabajador será citado mediante un correo electrónico para que participe. La primera parte de las jornadas se hará de forma conjunta con todos los nuevos trabajadores. El gerente del centro realizará una pequeña presentación de la organización con información general: misión, visión, valores, servicios que se ofertan, beneficios sociales de los que puede disponer el trabajador... y se presentará a los mentores. Posteriormente se realizará una visita guiada por el centro, especialmente por las zonas comunes como el comedor, la sala de reuniones, la sala de archivos... y otras zonas que se consideren relevantes.

Tras esta visita, cada mentor se hará cargo de sus mentees y los presentará a sus respectivos equipos. Cada equipo realizará una dinámica de presentación denominada las salas multicine. Tras esta primera aproximación, se les deja tiempo a los trabajadores para que establezcan conversaciones informales. Para esto, una alternativa es que la jornada de bienvenida cuente con un almuerzo/comida preparada por la organización. Aunque no sea una actividad en sí misma, sí que proporciona un espacio distendido donde la gente puede empezar a conocerse y socializar.

Para finalizar, el mentor les proporcionará a los nuevos trabajadores el manual de acogida para que puedan consultar cualquier duda que les haya quedado. Este momento también es oportuno para firmar o completar documentos como la cesión de derechos de imagen, la realización de cuestionarios sobre las necesidades de formación y, por descontado, una encuesta de satisfacción con las jornadas.

\section{Talleres de mindfulness}

La realización de talleres de mindfulness también está enfocada a conseguir que los trabajadores experimenten indicadores fisiológicos de calma y control y, además, proporcionar herramientas para que puedan conseguir estos estados de relajación en un futuro, cuando se enfrenten a demandas en sus puestos de trabajo (Bishop et al. 2004). Hay una gran diversidad de estudios sobre mindfulness, así como otras técnicas contemplativas, que han mostrado la efectividad de este para reducir la ansiedad (Franco-Justo, Mañas-Mañas y Justo-Martínez 2009). Por otro lado, como demuestran los estudios de neurociencia, la práctica diaria de mindfulness puede producir cambios estructurales en el cerebro adulto (Hölzer et al. 2007). Estos datos son los precursores de otros estudios que demuestran la relación entre el mindfulness y el desempeño laboral (Chiesa, Calati y Serretti 2010), el engagement (Leroy, Anseel, Dimitrova y Sels 2013) y las emociones y los recursos positivos (Lyubomirsky y Layous 2013) entre otros.

Para ello se contará con un instructor profesional durante las dos primeras semanas y se dedicará la primera hora de la mañana al aprendizaje de esta técnica. Posteriormente, serán los mentores y los supervisores quienes deberán fomentar su práctica continuada. Una buena forma de incorporar el mindfulness en la vida cotidiana de los trabajadores es ofreciendo bien un lugar donde pueda realizarse sin distracciones o bien pautando momentos del día (por ejemplo, antes o después de los descansos para comer) donde el equipo pueda realizar esta práctica y, por supuesto, reforzar a aquellos que lo practiquen. 


\section{Resultados}

Se espera que la presente propuesta de intervención produzca un incremento de la autoeficacia laboral de los nuevos trabajadores y, a través de esta, se facilite la inserción en la organización, se incremente el bienestar y el engagement en el trabajo. Al igual que en otros constructos, como el capital psicológico positivo, se espera además que el hecho de trabajar simultáneamente las cuatro fuentes de la autoeficacia tenga un efecto sinérgico, incrementando la potencia de la intervención en términos de resultados positivos.

\section{Conclusiones}

Los beneficios del incremento de la autoeficacia en los empleados a nivel individual son evidentes, especialmente si pensamos que la mayoría de ellos, por su edad, cuentan con poca experiencia laboral y esta sea, quizá, su primera aproximación al mundo laboral. Con este programa se pretende paliar los efectos negativos de las demandas asociadas a la inserción en la organización, dotando a los trabajadores de recursos para hacerles frente. Por otro lado, el incremento del bienestar y el engagement derivado del aumento de la autoeficacia, supondrá también ganancias a nivel organizacional que, como se ha comentado en el apartado de evaluación, podemos medir en términos de rendimiento, productividad, desempeño... (Salanova, Breso y Schaufeli 2005). En un estudio realizado por Haro-García, en el que se calculó el retorno de la inversión (ROI) de un programa de coaching que aumentaba las creencias de autoeficacia, se produjo un retorno de seis veces el coste del programa, además de una reducción de la rotación del personal y un aumento de la atracción del talento (Haro-García 2012). Por otro lado, la autoeficacia, y el bienestar psicosocial derivado de esta, está relacionada con otros muchos constructos y resultados positivos que, aunque no estén contemplados como variable objetivo del presente estudio, se verán alterados por la intervención. Por ejemplo, según la hipótesis del happy-productive worker, los trabajadores con elevados niveles de bienestar psicosocial tienden a presentar un desempeño laboral elevado. Esta condición no solo afecta al propio trabajador sino que, además, suele transmitirse al equipo de trabajo, produciendo un incremento de la productividad grupal (Taris y Schreurs 2009).

El presente trabajo se ha centrado en aumentar los recursos personales del trabajador y es totalmente compatible con otro tipo de prácticas que aumenten también los recursos laborales. Todo ello contribuirá a generar resultados positivos para la persona, el grupo de trabajo y la organización en su conjunto. Además, puede sentar los cimientos para que la organización se transforme en una HERO (HEalthy and Resilient Organization). Una HERO se define como «una organización que desarrolla esfuerzos sistemáticos, planificados y proactivos para mejorar su salud psicosocial y financiera, mediante prácticas saludables y recursos para mejorar a nivel de tareas, ambiente social y organizacional, especialmente en situaciones de crisis y cambios bruscos» (Salanova, Llorens, Cifre y Martínez 2012).

\section{Referencias bibliográficas}

Bandura, Albert. 2001. «Social cognitive theory: an agentic perspective». En Nuevos horizontes en la investigación sobre la autoeficacia, ed. María Luisa Salanova, Rosa María Grau, Isabel María Martínez, Eva Cifre, Susana Llorens y Mónica García-Renedo (178-204). Castellón: Publicacions de la Universitat Jaume I. 
Bersin Deloitte's. 2014. Onboarding Software Solutions 2014: On-Ramp for Employee Success.

Bishop, Scott R., Mark A. Lau, Shauna Shapiro, Linda E. Carlson, Nicole D. Anderson, James Carmody, Zindel V. Segal, Susan Abbey, Michael Speca, Drew Velting y Gerald Devins. 2004. «Mindfulness: a proposed operational definition». Clinical Psychology: Science and Practice 11(3): 230-241.

Blanch, Joseph M., Miguel Ángel Sahagún, Leonor M. Cantera y Genís Cervantes. 2010. "Cuestionario de Bienestar Laboral General: estructura y propiedades psicométricas». Revista de Psicología del Trabajo y de las Organizaciones 2(2): 157-170.

Calderón, José Luis, Francisco A. Laca y Manuel Pando. 2017. «La autoeficacia como mediador entre el estrés laboral y el bienestar». Psicología y salud 27(1): 7178.

Centro Canadiense de Seguridad y Salud Ocupacional (CCSSO). 2002. Bienestar en el lugar de trabajo. Recuperado de: http://www.ccsso.ca/oshanswers/hsprograms/job_design.html [Consultado el 19 de agosto de 2019].

Chiesa, Alberto, Raffaella Calati y Alessandro Serretti. 2010. «Does mindfulness training improve cognitive abilities? A systematic review of neuropsychological findings». Clinical Psychology Review 31(3): 449-464.

Consiglio, Chiara, Lara Borgogni, Guido Alessandri y Wilmar B. Schaufeli. 2013. «Does self-efficacy matter for burnout and sickness absenteeism? The mediating role of demands and resources at the individual and team levels». Work \& Stress: An International Journal of Work, Health \& Organisations 27(1): 22-42.

De Miguel, María Luisa, Sandra Dema y Susana Pérez. 2010. El mentoring como práctica de intervención para la mejora de los procesos de capacitación y socialización de las empresarias noveles. Tesis de maestría. Oviedo: Universidad de Oviedo.

Fisher, Cynthia D. 1986. «Organizational socialization: an integrative perspective». Research in Personnel and Human Resource Management 4: 101-145.

Franco-Justo, Clemente, Israel Mañas-Mañas y Eduardo Justo-Martínez. 2009. «Reducción de los niveles de estrés, ansiedad y depresión en docentes de educación especial a través de un programa de mindfulness». Revista Educación Inclusiva 2(3): 11- 33.

Haro-García, José Manuel. 2012. «El análisis de los resultados del coaching ejecutivo: una propuesta de clasificación». Papeles del Psicólogo 33(3): 221-226.

Hölzer, Britta K., Ulrich Ott, Hannes Hempel, Andrea Hackl, Katharina Wolf, Rudolf Stark y Dieter Vaitl. 2007. «Differential engagement of anterior cingulate and adjacent medial frontal cortex in adept meditators and non-meditators». Neuroscience Letters 421(1): 16-21.

Jones, Gareth. 1986. «Socialization tactics, self- efficacy, and newcomers' adjustments to organizations». Academy of Management Journal 29(2): 262-279.

Leroy, Hannes, Frederik Anseel, Nicoletta G. Dimitrova y Luc Sels. 2013. «Mindfulness, authentic functioning and work engagement: a growth modeling approach». Journal of Vocational Behavior 82: 238-247.

Lisbona, Ana, Francisco José Palací, Marisa Salanova y Michael Frese. 2018. «The effects of work engagement and self-efficacy on personal initiative and performance». Psicothema 30(1): 89-96.

Lyubomirsky, Sonja y Kristin Layous. 2013. «How do simple positive activities increase well-being?». Psychological Science 22(1): 57-62.

Maffei, Luis, Carlos Spontón, Marcos Spontón, Estanislao Castellano y Leonardo Adrián Medrano. 2012. «Adaptación del Cuestionario de Autoeficacia Profesional (AU-10) a trabajadores cordobeses». Pensamiento Psicológico 10(1): 51-62. 
Martínez-Martínez, Isabel y Marisa Salanova-Soria. 2006. «Autoeficacia en el trabajo: el poder de creer que tú puedes». Estudios financieros, 279: 175-202.

Mendoza, Roberto. 2003. "¿Sabe cuánto le cuesta la rotación de personal?». Revista Escuela de Administración de Negocios 48: 104-109.

Núñez, Pedro. 2010. Los efectos de la formación y el desarrollo de los recursos humanos en las empresas familiares españolas. Tesis doctoral. Jaén: Universidad de Jaén.

Taris, Toon y Paul J. G. Schreus. 2009. «Well-being and organizational performance: an organizational-level test of the happy-productive worker hypothesis». Work \& Stress 23(2): 120-136

Tena, Gloria. 2002. «El contrato psicológico: relación laboral empresa-trabajador». Acciones e Investigaciones Sociales 15: 85-107.

Tristán, Raúl. 2017. «Mentoring: el antiguo oficio de acompañar compartiendo conocimientos». Eleconomista.es. Recuperado de: http://www.eleconomista.es/firmas/noticias/8504925/07/17/Mentoring-elantiguo-oficio-de-acompanar-compartiendo-conocimientos.html [Consultado el 4 de agosto de 2019].

Reyes, Yolanda e Inocencia Martínez. 2007. «Los contratos psicológicos: sus efectos en los resultados de las organizaciones». En V Encuentro Iberoamericano de Finanzas y Sistemas de Información (EFSI). Alicante: Universidad de Alicante

Robinson, Sandra L. y Denise M. Rousseau. 1994. "Violating the psychological contract: not the exception but the norm». Journal of Organizational Behaviour 15: $245-259$

Salanova, Marisa, Edgar Breso y Wilmar B. Schaufeli. 2005. «Hacia un modelo espiral de las creencias de eficacia en el estudio del burnout y del engagement». Ansiedad y Estrés 11(2-3): 215-231.

Salanova, Marisa, Susana Llorens y Wilmar B. Schaufeli. 2011. "Yes, I Can, I Feel Good, and I Just Do It! On Gain Cycles and Spirals of Efficacy Beliefs, Affect and Engagement». Applied Psychology 60(2): 255-285.

Salanova, Marisa, Susana Llorens, Eva Cifre e Isabel M. Martínez. 2012. "We need a Hero! Towards a validation of the healthy \& resilient organization (HERO) model». Group \& Organization Management 37(6): 785-822.

Schaufeli, Wilmar y Arnold Bakker. 2003. Utrecht Work Engagement Scale (UWES) Preliminary Manual. Utrecht: Occupational Health Psychology Unit Utrecht University.

Schneider, Benjamin. 1987. "The people make the place». Personnel Psychology 40: 437-453.

Peiró, José María y Fernando Prieto (eds.). 1996. Tratado de Psicología del Trabajo. Vol. I: Actividad Laboral en su contexto. Madrid: Ed. Síntesis

Pérez-Fuentes, María del Carmen, María del Mar Molero, Ana Belén Barragán, África Martos, María del Mar Simón y José Jesús Gázquez. 2018. «Autoeficacia y Engagement en estudiantes de Ciencias de la Salud y su relación con la autoestima». Publicaciones 48(1): 161-172.

Wood, Robert E. y Albert Bandura. 1989. «Impact of Conceptions of Abitily on SelfRegulatory Mechanisms and Complex Decision Making». Journal of Personality and Social Psychology 56(3): 407- 415. 